\title{
La transparencia y el interés público en el arbitraje de inversiones*
}

\author{
Transparency and Public Interest in Investment Arbitration
}

Nayiber Febles Pozo ${ }^{\mathrm{a}}$

Universidad Internacional de Valencia, España

feblespozo@gmail.com

DOI: https://doi.org/10.11144/Javeriana.vj69.tiai

Recibido: 02 Septiembre 2019

Aceptado: 30 Septiembre 2019

Publicado: 15 Agosto 2020

\section{Resumen:}

Resulta paradójico que las diferentes normas institucionales y arbitrales de que disponen las partes en litigio hayan sido tradicionalmente escasas con respecto a su transparencia procesal, al dejar, en gran medida, esas cuestiones a la discreción de las partes, o, a falta de acuerdo, al tribunal arbitral. Los problemas de procedimiento en el arbitraje internacional son cada día más frecuentes, de mayor complejidad y de máxima preocupación. Cuestiones estas que, junto al interés público, son objeto de interés en el presente artículo.

Palabras clave: transparencia, arbitraje internacional, interés público.

\section{Abstract:}

Paradoxically, the different institutional and arbitration rules available to the parties in dispute have traditionally been scarce regarding procedural transparency, leaving these issues mainly to the discretion of the parties, or, in the absence of agreement, to the arbitral tribunal. Procedural problems in international arbitration are increasingly frequent, of greater complexity and utmost concern. These are issues that, together with the public interest, are an object of interest in this article.

Keywords: transparency, international arbitration, public interest.

\section{Introducción}

En el ámbito del arbitraje de inversiones el argumento principal para una mayor exigencia de transparencia y participación pública es la presencia del interés público, teniendo en cuenta que el arbitraje de inversión tiene una fuerte influencia en el erario, es decir, en las finanzas públicas de los Estados, con los que debe hacer frente a las altas cuantías exigidas por los inversores. En este sentido, se justifica la gran preocupación de la sociedad por la transparencia en los procedimientos arbitrales y los efectos que puede provocar en los presupuestos estatales, frutos de la recaudación de los impuestos fiscales pagados por la sociedad ${ }^{1}$.

En el ámbito del derecho internacional, y del propio derecho internacional de las inversiones, resulta necesario que la transparencia esté presente en importantes elementos o cuestiones procesales relevantes para el Investor-State Dispute Settlement (ISDS de ahora en adelante), como pueden ser el acceso a las audiencias, el conocimiento de las presentaciones tanto escritas como orales de las partes y de las resoluciones finales; es decir, el conocimiento público del Laudo que resuelve la disputa. Todo ello, ante la argumentación de que la transparencia en el contexto de la solución de controversias inversor-Estado es un término generalmente indefinido $^{2}$, y a los efectos de esta investigación, constituyen el fundamento principal para distinguir dos elementos procesales en los que la aplicación de la transparencia es un factor clave para el ISDS: (i) la publicación de la información y los documentos presentados al inicio y durante el procedimiento y (ii) la participación de terceros y el acceso público a las audiencias durante el procedimiento.

Notas de autor

a Autor de correspondencia. Correo electrónico: feblespozo@gmail.com 


\section{La transparencia en el procedimiento arbitral: el acceso a la información durante el proceso: generalidades}

En ocasiones, la información sobre los procedimientos arbitrales puede ser de dominio público a pesar de la ausencia de un requisito formal de transparencia ${ }^{3}$. Esto puede suceder, por ejemplo, en la presentación del laudo a un tribunal nacional para llevar a cabo el reconocimiento y la ejecución del mismo, lo que permite el conocimiento judicial de los hechos acaecidos durante el procedimiento arbitral y, en determinadas circunstancias, el laudo puede convertirse en un documento público como cualquier otro presentado ante un procedimiento judicial nacional ${ }^{4}$.

Respecto a las alegaciones de las partes, si bien las Reglas del Mecanismo Complementario del Centro Internacional de Arreglos de Diferencias Relativas a Inversiones (CIADI) no obligan ni prohíben la divulgación de las alegaciones de las partes, resulta muy significativo el caso Piero Foresti vs. Sudáfrica ${ }^{5}$, que atrae un amplio interés de la sociedad civil. En el presente caso, Piero Foresti y otras nueve personas con distintas nacionalidades (los demandantes) presentan demanda contra la República de Sudáfrica en base a los Acuerdos de Promoción y Protección Recíprocas de Inversiones (APPRI) entre Italia y Sudáfrica de 1997, y el APPRI entre Sudáfrica y la Unión Económica Belgo-Luxemburgo de 1998 sobre la protección recíproca de las inversiones. El procedimiento arbitral se administró bajo las reglas del CIADI. Los demandantes impugnaron la Ley de Recursos Minerales y de Petróleo, promulgada por Sudáfrica por razones de política pública. La parte demandada alega que la ley fue promulgada para proteger cuestiones que afectan directamente a los ciudadanos: derechos humanos, medio ambiente y desarrollo sostenible.

Por la importancia del caso, varias Organizaciones no Gubernamentales (ONGs) solicitaron al tribunal presentar sus escritos. Admitidas como partes no contendientes (NDP por sus siglas en inglés) en el procedimiento, el tribunal ordenó a las partes litigantes que revelaran sus alegatos escritos a cinco peticionarios de amicus no gubernamentales, teniendo en cuenta dos principios básicos: i) que la intención de permitir la participación de las partes no contendientes es que proporcionan información útil y ii) permitir que las Partes no contendientes tengan acceso a los documentos presentados por las partes en el procedimiento, incluso, en contra de las objeciones de los reclamantes, para permitir que las partes no contendientes puedan centrar sus escritos en las cuestiones relacionadas con el caso ${ }^{6}$.

Ahora bien, aun y cuando las partes demostraron su disconformidad en revelar la información solicitada por los amicus admitidos en el procedimiento, el conocimiento público de dicha información no llegó a realizarse, debido a que los demandantes solicitaron la interrupción del procedimiento teniendo en cuenta el Acuerdo de Compensación firmado posteriormente con la parte demandada en el que se les otorgaron nuevos derechos mineros ${ }^{7}$. Además, argumentaron que dicho acuerdo fue considerado como una respuesta o satisfacción parcial a sus pretensiones y que, en relación con los costos del arbitraje y las condiciones económicas actuales, era más apropiado finalizar el procedimiento ${ }^{8}$. La parte demandada, en respuesta a la solicitud de terminación del procedimiento presentado por los demandantes, expresó su desacuerdo, debido a que los demandantes habían solicitado la terminación del procedimiento "sin perjuicio", es decir, sin la autoridad de cosa juzgada, dejando la posibilidad de que los demandantes pudieran presentar las mismas reclamaciones en fechas posteriores.

Como consecuencia, el tribunal ordenó continuar las actuaciones 9 . Después de que los demandantes aceptaran la terminación del procedimiento y "la emisión de un laudo que desestimara sus reclamaciones con efecto de cosa juzgada"10, el Tribunal decide finalmente dar por finalizado el procedimiento arbitral, condenando a los demandantes a indemnizar a la parte demandada por los gastos incurridos durante el procedimiento y desestimar sus reclamaciones ${ }^{11}$. Este caso demuestra que mientras no existan normas vinculantes que establezcan la obligatoriedad en la publicación de la información durante el proceso, la 
publicidad de las actuaciones seguirá resultando escasa y, muy rara vez, resultará efectiva. En este sentido, puede observarse que la divulgación de las alegaciones de las partes así como algunas fases del procedimiento siguen carentes de transparencia.

En cuanto a los problemas relacionados con el uso y la accesibilidad de la información durante el procedimiento, actualmente están siendo parcialmente abordados. La Secretaría del CIADI publica un informe semestral ${ }^{12}$ en el que se detallan las estadísticas de sus actividades, incluidos los casos registrados y administrados, las fuentes de reclamaciones de los inversores y los Estados, la distribución regional y sectorial de las reclamaciones, los países de origen de los árbitros y los resultados de las controversias, lo que lo vuelve el más completo de los informes compilados institucionalmente. Sin embargo, omite puntos clave de interés que resultan muy importantes en cuanto al conocimiento público de la información, como pueden ser la duración de los procedimientos, las tasas administrativas, los costos del tribunal, la publicación de los documentos, los alegatos presentados y las cantidades reclamadas y concedidas.

El acceso a la información puede servir como medio para asegurar una mayor legitimidad del sistema arbitral. El ISDS, por su propia característica de descentralización del sistema, permite que la transparencia no se centre solo en cuestiones de acceso al procedimiento, más bien la transparencia sirve como un medio para desafiar prácticas tan diversas que contribuyen involuntariamente a una falta de la misma, como puede ser la persistencia en no publicar los laudos, la participación de las secretarías arbitrales en el proceso interno, la acumulación de la información confidencial o experiencia especializada de ciertas empresas que no se divulgan públicamente. $\mathrm{Al}$ respecto, la transparencia también puede servir como un reconocimiento pragmático de que la información es oscurecida al público por el secreto y la confidencialidad, y requiere una sistematización para ser comprensible.

La transparencia ayuda a exponer ante el público el funcionamiento interno del sistema. La aprobación del Reglamento de Transparencia de la CNUDMI sobre arbitraje inversor-Estado ha servido para implementar muchas de estas preocupaciones en un conjunto de directrices unificadas, las cuales contienen disposiciones sobre el ejercicio del poder discrecional por parte de un tribunal arbitral. Este tribunal opera bajo las siguientes obligaciones: la obligación de tener en cuenta el interés público en la transparencia ${ }^{13}$, las obligaciones de publicación de documentos en diferentes etapas de los procedimientos arbitrales ${ }^{14}$ y la obligación de llevar a cabo audiencias en público ${ }^{15}$.

Sin embargo, una cuestión relativa a la transparencia procesal en el arbitraje internacional merece ser analizada: el interés público en el arbitraje internacional, con especial énfasis en el arbitraje de inversiones. Con frecuencia suelen encontrarse referencias al interés público en el arbitraje de inversiones como forma de posibilitar una mayor transparencia en el procedimiento arbitral, pero, aunque muchos conflictos de inversión tienen una implicación importante en temas que merecen la atención del público, el concepto de interés público sigue siendo indeterminado, impreciso y vago. Por lo que cabría preguntarse ¿qué constituye el interés público en el arbitraje internacional?

\section{De intereses privados a interés público en el arbitraje internacional ¿Por qué abrir la participación social en el arbitraje de inversiones?}

La cuestión del interés público con respecto a la transparencia del arbitraje puede ser tratada desde dos vertientes distintas. La primera, está relacionada con el interés general del público en conocer y ser informado de aquellos asuntos que revisten importancia para la sociedad, así como el actuar de los tribunales de inversión respecto a la revisión de las acciones soberanas de los Estados partes en el procedimiento arbitral ${ }^{16}$. La segunda vertiente se corresponde al interés público con respecto procedimiento arbitral, en relación a si una tercera parte, además del Estado parte en el procedimiento, puede representar tal interés público en el 
arbitraje. Al respecto, el debate académico y la jurisprudencia sobre el tema, desde el punto de vista práctico, principalmente se han centrado en cuestiones relativas a la representación de los intereses públicos a través de las comunicaciones de amicus curiae.

Es evidente que el interés público es una de las principales peculiaridades de las controversias de inversión que las distingue de los conflictos que surgen del arbitraje comercial internacional entre partes privadas. En teoría, el interés público puede referirse, por una parte, al interés de un Estado y sus mandantes y, por otra, a cuestiones que abarcan los intereses comunes de la humanidad, como las preocupaciones medioambientales, de Salud Pública y de Derechos humanos ${ }^{17}$. Sin embargo, en el ámbito normativo, las disposiciones legales pertinentes carecen de una referencia directa al término y a la propia conceptualización del interés público. Esto ha propiciado que, en la práctica, el concepto de interés público se haya ido elaborando con el transcurso del tiempo por los propios tribunales arbitrales en una serie de disputas de inversiones importantes, las cuales han ido estableciendo ciertas pautas o elementos que condicionan la propia existencia del concepto de interés público en este tipo de disputas, tal y como sucedió en el caso Methanex vs. Estados Unidos de América ${ }^{18}$. Cabe pues, abrir un paréntesis para adentrarnos en las particularidades del caso.

El caso citado tuvo como objeto una disputa de inversión entre Methanex Corporation, con sede en Canadá, y los Estados Unidos, en base a las disposiciones del Capítulo 11 del Tratado de Libre Comercio de América del Norte (TLCAN) sobre inversiones. La Demandante (Methanex Corporation) es una empresa productora, transportista y comercializadora de Metanol, un componente clave del Methyl Tertiary Butyl Ether (MTBE) sustancia química que se usa para aumentar el contenido de oxígeno y actuar como un potenciador del octano en la gasolina sin plomo. La parte demandante, en desacuerdo con la orden del Estado de California de marzo de 1999 de prohibir el uso de MTBE a finales de 2002, decide comenzar un procedimiento arbitral contra los Estados Unidos. El Estado de California argumentó que la medida tomada para prohibir el uso del MTBE era necesaria, teniendo en cuenta que dicha sustancia contaminó los suministros de agua potable, lo que representa un riesgo considerable para la salud, la seguridad de las personas y el medio ambiente. Methanex Corporation alega que la medida tomada por el Gobierno de California no era efectiva, y que el incumplimiento de las leyes ambientales y la Clean Water Act of the United States fue lo que provocó la presencia de MTBE en los suministros de agua de California. La parte demandante argumentó también que la prohibición impuesta por el Gobierno de California era equivalente a una expropiación de su inversión y, por tanto, se incumplió el Art. 1110 del TLCAN, así como la existencia de una violación del deber de trato nacional establecido en el Art. 1102 del TLCAN y un incumplimiento de las obligaciones mínimas reconocidas en los estándares internacionales según el Art. 1105 del propio TLCAN. Por todo ello, la Demandante exigía a EE.UU. una indemnización por daños ascendente aproximadamente a 1 Billón de dólares.

Las cuestiones planteadas en el caso sobre la interacción entre la protección del inversor y la protección del medio ambiente fueron tan agudas, que dieron motivos para que el International Institute for Sustainable Development (IISD) solicitara al tribunal arbitral presentar un escrito como amicus curiae relacionado con asuntos legales críticos que involucran el interés público presente en el caso, hacer presentaciones orales para respaldar su pretensión con el escrito de amicus en el momento adecuado del procedimiento y tener estatus de observador en las audiencias orales ${ }^{19}$. También presentó solicitud para participar como amicus Communities for a Better Environment and the Earth Island Institute, entre otros.

La parte demandante se opuso a las peticiones de participación como amicus realizadas, alegando principalmente cuestiones relacionadas con la confidencialidad. El tribunal, en audiencia posterior, admite las solicitudes y, por ende, la participación como amicus de las peticionarias, admitiendo también que los Gobiernos de Canadá y México presentaran sus alegatos ${ }^{20}$. Una vez admitida la participación del IISD como amicus, éste presenta su escrito en el que solicita al tribunal arbitral que se reconozca la fuerte presencia del interés público presente en el litigio, que lo hace diferente del interés comercial privado que normalmente suele estar presente en el arbitraje. En este sentido, el escrito presentado reflejó la situación provocada por 
el MTBE en el saneamiento de agua potable en el Estado afectado y las graves consecuencias que traería para la población en el futuro, así como las intenciones que motivaron al Gobierno de California al tomar la medida con el objetivo de contrarrestar los nocivos efectos ocasionados por el uso del MTBE. Además, trató temas de gran relevancia relacionados con el desarrollo sostenible y recogió una definición de los conceptos de expropiación y trato nacional.

Después de una extensa revisión y un profundo análisis de los hechos y los documentos presentados en el procedimiento, y en respuesta a los escritos presentados por los amicus, el tribunal admite la existencia de un interés público en la disputa, no solo por la presencia del Estado como parte en el litigio, sino por el controvertido objeto de la disputa. De este modo, el tribunal expresó:

indudablemente existe un interés público en este arbitraje. Las cuestiones sustantivas se extienden mucho más allá de las planteadas por el habitual arbitraje transnacional entre las partes comerciales. Esto no se debe simplemente a que una de las partes en litigio es un Estado: por supuesto, hay disputas que involucran a Estados que no tienen mayor interés público general que un conflicto entre particulares. El interés público en este arbitraje surge de su objeto, tal como se sugiere fuertemente en las peticiones $(. . .)^{21}$.

Otro ejemplo de gran relevancia es el caso Suez-Vivendi y otros vs. República Argentina ${ }^{22}$. Los demandantes (Aguas Argentinas S. A. (AASA), Suez, Sociedad General de Aguas de Barcelona S. A. (AGBAR), Vivendi Universal S. A. (Vivendi) y AWG Group Ltd. (AWG)) presentaron una solicitud de arbitraje ante el CIADI contra la República de Argentina, alegando una serie de supuestos actos y omisiones del Estado, incluyendo el supuesto incumplimiento o negativa a aplicar ajustes previamente convenidos a los mecanismos de cálculo y ajuste de tarifas, lo que provocó ciertas afectaciones a las inversiones de los demandantes en una concesión obtenida para el suministro de agua potable y desagües cloacales en la Ciudad de Buenos Aires y algunos municipios circundantes. Al respecto, los demandantes invocaron el consentimiento otorgado por la parte demandada para solucionar las diferencias ante el CIADI en virtud de los APPRI entre Francia y la República Argentina de 1991, entre la República Argentina y el Reino de España de 1991 y entre la República Argentina y el Reino Unido de Gran Bretaña e Irlanda del Norte de 1990, teniendo en cuenta las distintas nacionalidades de los demandantes ${ }^{23}$.

Ante las complejidades del caso por las cuestiones de derecho público presente, cinco ONGs - los peticionarios- (Asociación Civil por la Igualdad y la Justicia (ACIJ), Centro de Estudios Legales y Sociales (CELS), Centro para el Derecho Internacional Ambiental (CIEL), Consumidores Libres Cooperativa Lda. de Provisión de Servicios de Acción Comunitaria, y la Unión de Usuarios y Consumidores), presentaron ante el CIADI una "Petición de Transparencia y Participación en Calidad de Amicus Curiae". Dicha petición fue fundamentada teniendo en cuenta las cuestiones de interés público y derechos fundamentales presentes en el caso. La petición dirigida al tribunal tuvo como principal objeto tres cuestiones esenciales. La primera, que el tribunal les concediera el acceso a las audiencias, efectuar presentaciones orales y que las audiencias fueran abiertas al público, citando el caso Methanex vs. Estados Unidos de América comentado antes; la segunda, tener oportunidad suficiente para poder exponer sus argumentos legales en carácter de amicus curiae; y, en tercer lugar, tener acceso oportuno, suficiente e irrestricto a los documentos del proceso ${ }^{24}$. Una vez trasladada dicha solicitud por el tribunal a las partes en litigo para que se pronunciaran al respecto, los demandantes se opusieron mientras que la parte demandada manifestó su conformidad.

Analizada las peticiones realizada por las ONGs, el tribunal las admite en parte. Decide denegar la solicitud de acceso y asistir a las audiencias del caso, pero considera que el caso es apropiado para que personas idóneas que no sean partes en el mismo puedan realizar presentaciones de amicus curiae. Así pues, una vez autorizada la presentación de escrito del amicus por el tribunal, también decidió sobre la solicitud de acceso a la documentación formulada por los peticionarios. Así pues, tras examinar las cuestiones planteadas en el caso y, especialmente, en lo relacionado al interés público, el tribunal concluyó que el caso involucraba potencialmente cuestiones de interés público: 
el factor que da a este caso interés público particular, es que la disputa de inversión se centra en los sistemas de distribución y alcantarillado de un área metropolitana mayor, la Ciudad de Buenos Aires y sus Municipios alrededores. Esos sistemas proporcionan servicios públicos básicos a millones de personas y, como resultado, pueden plantear una variedad de cuestiones complejas de Derecho público e internacional, incluidas las consideraciones de Derechos humanos. Cualquier decisión dictada en este caso, ya sea a favor de los Demandantes o del Demandado, tiene el potencial de afectar el funcionamiento de esos sistemas y, por lo tanto, del público al que sirven 25 .

En este sentido, el propio tribunal, en sus consideraciones, ha dejado claro aquellos aspectos presentes en la disputa y que hacen que en la misma exista presencia de un interés público.

Consideramos que ambos casos ejemplifican aquellos factores que identifican la presencia de un interés público en el arbitraje de inversiones, como pueden ser la presencia estatal, el objeto de la litis, es decir, la actividad inversora, cuando involucre cuestiones de derecho público y cuestiones de políticas públicas, y que son llevadas a cabo por los Estados en salvaguarda del interés de la sociedad y la propia responsabilidad internacional de los Estados en contraposición a la responsabilidad de las empresas que emana del derecho privado.

Sin embargo, estos elementos no constituyen una lista exclusiva, por lo que un tribunal puede determinar la existencia de interés público basado en criterios distintos ${ }^{26}$, tal y como sucedió un año más tarde en el caso Aguas Provinciales de Santa Fe vs. Argentina ${ }^{27}$ un año más tarde. Este caso tuvo idéntica similitud con el caso Suez-Vivendi vs. Argentina comentado antes. Los demandantes (Suez, Sociedad General de Aguas de Barcelona, S. A. (“AGBAR”), Aguas Provinciales de Santa Fe S. A. (“APSF”), e InterAguas Servicios Integrales del Agua, S. A.), presentaron semejante solicitud al caso Suez-Vivendi ante el CIADI para iniciar el procedimiento arbitral. Esta se refería a las inversiones de los demandantes en una concesión para el suministro de agua potable y desagües cloacales en la provincia argentina de Santa Fe y una serie de supuestos actos y omisiones de Argentina, incluyendo el supuesto incumplimiento o negativa a aplicar ajustes previamente convenidos a los mecanismos de cálculo y ajuste de tarifa.

En el presente caso, se presentó también una petición de amicus curiae, esta vez por una ONGs (Fundación para el Desarrollo Sustentable) y tres personas naturales expertas en leyes, derechos humanos y desarrollo (peticionarios). En dicha petición, los peticionarios exigieron:

Solicitamos que dicha participación como amicus curiae pueda tener acceso a la información que consta en el proceso, y de participar de los actos futuros del procedimiento con el fin de poder manifestar, mediante presentaciones por escrito (amicus curiae brief) y exposiciones orales en las audiencias, nuestros puntos de vista que permitirán acercar mayor transparencia al proceso y al mismo tiempo brindar al Tribunal una perspectiva enriquecedora y distinta a la de las partes ${ }^{28}$.

Una vez que el tribunal dio traslado de la petición de amicus curiae realizada por los terceros a las partes en litigio, fue aceptada por la parte demandada, pero los demandantes presentaron su rechazo a la misma.

Sin embargo, el tribunal decide admitir en parte la petición de participación como amicus de los peticionarios. En respuesta a la misma, puede apreciarse que el tribunal ofreció, en comparación con el caso anterior, una definición más amplia del interés público, a través de la cual la intervención de terceros se ha ido admitiendo por los tribunales en los casos que involucran cuestiones de interés público, al expresar: "porque las decisiones en estos casos tienen el potencial, indirectamente, de afectar a personas más allá de las que están inmediatamente involucradas como partes en el caso" ${ }^{29}$. Una vez examinadas todas las cuestiones planteadas por las partes en el procedimiento, el Tribunal concluye que el caso involucra potencialmente cuestiones de interés público. Entonces, siguiendo un enfoque similar al caso Suez-Vivendi, el tribunal subrayó:

el factor que otorga a este caso interés público particular, es que la disputa de inversión se centra en los sistemas de distribución de agua y alcantarillado de las áreas urbanas de la provincia de Santa Fe. Esos sistemas proporcionan servicios públicos básicos a cientos de miles de personas y, como resultado, pueden plantear una variedad de cuestiones complejas de Derecho público e internacional, incluidas las consideraciones de Derechos humanos. Cualquier decisión dictada en este caso, ya sea a favor de los Demandantes o del Demandado, tiene el potencial de afectar el funcionamiento de esos sistemas y, por lo tanto, del público al que sirven ${ }^{30}$. 
Los casos hasta aquí comentados, entre otros, son un ejemplo de la existencia de una inexcusable presencia del interés público en las disputas inversor-Estado. Además, aunque para el análisis del interés público en el arbitraje de inversiones son varios los aspectos a tener en cuenta, consideramos que el mismo puede ser fácilmente identificable a partir de dos contextos relevantes en este tipo de disputas, como son:

i. El desafio a medidas gubernamentales: las disputas inversor-Estado implican desafíos por parte del inversor al ejercicio del poder público estatal. No solo respecto al tratamiento del Estado del inversor en particular, sino el impacto en el inversor del ejercicio más general del poder regulador del Estado ${ }^{31}$, lo que la hace la opción más frecuente.

ii. El desarrollo de un derecho internacional de inversiones: en el cual la transparencia sea un factor fortalecedor del propio derecho internacional de las inversiones y que posibilite un mayor conocimiento público de las decisiones arbitrales como condición previa para la creación y evolución de una jurisprudencia indeleble, lo que garantiza preservar los intereses de las partes: Estado e inversor. Además, se facilitaría el desarrollo de normas sustantivas del derecho de inversiones a través de la práctica arbitral y un mayor conocimiento público de los tratados firmados por los Estados. En este sentido, consideramos que si tanto interés tiene el inversor en conocer sus derechos y la protección que disfruta en el Estado extranjero, así como los Estados receptores de la inversión respecto de los inversores extranjeros que reciben, los ciudadanos también tienen el derecho de informarse sobre los APPRI que han firmado sus Estados, y de saber cómo el alcance o el precio de los poderes reguladores de sus gobiernos podrían verse afectados por la celebración de los propios tratados ${ }^{32}$. Además, debe tenerse en cuenta, que en la inmensa mayoría de los arbitrajes de inversiones existen siempre consideraciones de derechos fundamentales: el objeto de la inversión en gran medida afecta directa o indirectamente un bien público.

Lo expresado hasta aquí es más que suficiente para demostrar la existencia de un interés público determinable, identificable, múltiple y heterogéneo en el arbitraje inversor-Estado en el marco de un tratado internacional. Dicho interés público no solo está presente en el arbitraje de inversiones, sino también en el arbitraje comercial internacional cuando existe presencia de un Estado o entidades públicas, estatales, que puede implicar asuntos esenciales de políticas públicas que tengan un impacto en los fondos públicos y elevar las obligaciones internacionales de un Estado ${ }^{33}$.

Tanto los Estados como sus propias instituciones o entidades públicas deben actuar con mucha prudencia y precaución en el ámbito del arbitraje internacional. Algunos casos de arbitraje inversor-Estado han tenido como causa fundamental objeto de la litis, violaciones de los tratados como consecuencia de presuntas infracciones del contrato por parte de los Estados o entidades estatales ${ }^{34}$, en ocasiones por supuestas actuaciones fraudulentas ${ }^{35}$, al existir una implicación de los gobiernos o Estados demandados como parte de la relación jurídica en la que hay presente importantes cuestiones de derecho internacional. Por ejemplo, tal y como sucedió en el caso World Duty Free Company Ltd. vs. Kenia ${ }^{36}$, caso administrado bajo las Reglas del CIADI y que se basó en el incumplimiento de un contrato.

En 1989, el presidente de World Duty Free mostró su interés en obtener la concesión para la construcción, mantenimiento y operación de un complejo de tiendas libres de impuestos en los aeropuertos internacionales de Nairobi y Mombassa, Kenia. Su socio keniano le recomendó que hiciera la solicitud directamente al presidente de la República de Kenia, su Excelencia Daniel Moi. Para ello, ambas partes sostuvieron un encuentro en la residencia presidencial del Sr. Moi, en el que los representantes de World Duty Free, además de presentar su propuesta, hacen entrega de una carpeta con dos millones de dólares al asistente del presidente keniano el Sr. Sajjad. Una vez terminado el encuentro, el Sr. Sajjad manifestó estar contento por el encuentro realizado y, posteriormente, el presidente de la República keniana el Sr. Moi ordenó que se le concediera a dicha compañía el contrato de tiendas de libres impuestos. 
Firmado el contrato por la compañía World Duty Free, se invierte una considerable suma de dinero durante la ejecución del proyecto. Sin embargo, años después surgen problemas entre las partes, problemas a los que el Gobierno keniano reacciona con la nulidad del contrato una vez que tuvo conocimiento de que se obtuvo mediante prácticas fraudulentas, a través de pagos corruptos. Como consecuencia, World Duty Free inició un procedimiento de arbitraje contra el Estado de Kenia alegando incumplimiento del contrato, y reclamando la devolución de las tiendas o una indemnización por un importe de 500 millones de dólares. Kenia alega, que como cuestión de política pública internacional y en cumplimiento de las leyes kenianas e inglesas - aplicables al contrato-, el contrato entre las partes era ilegal y, por tanto, las reclamaciones de World Duty Free deben ser rechazadas. En este sentido, el tribunal arbitral desestimó la demanda interpuesta por la compañía World Duty Free, al considerar que la corrupción es contraria al orden público internacional, motivo por el cual, aceptó la anulación del contrato solicitada por la parte demandada y, de esta manera, niega a la parte demandante legitimación activa por haber cometido una violación del orden público internacional.

Otro ejemplo muy peculiar es el caso de las Empresas Lucchetti, S. A. (Chile) y Lucchetti Perú, S. A. (Perú) vs. República del Perú ${ }^{37}$. La controversia se refirió a la construcción de una fábrica de pastas en la Municipalidad de Lima, y fue planteada al CIADI en el marco de su propio convenio. Las partes demandantes (Empresas Lucchetti, S. A. (Chile) y Lucchetti Perú, S. A.) invocaron las disposiciones sobre solución de controversias del APPRI entre el Gobierno de la República de Chile y el Gobierno de la República del Perú para la promoción y protección recíproca de las inversiones ${ }^{38}$. Las partes demandantes sostienen que todas las autorizaciones y permisos administrativos y municipales necesarios para la construcción de la fábrica industrial los obtuvieron conforme a las leyes, reglamentos y prácticas habituales en el Perú. Sin embargo, a finales de 1997 e inicios de 1998, la Municipalidad Metropolitana de Lima (Municipalidad de Lima) anuló los permisos otorgados a la segunda demandante (Lucchetti Perú, S. A.) para la construcción de su fábrica, invocando problemas ambientales y supuestos vicios de los actos de otorgamiento de los permisos. Todos los actos de anulación de los permisos y sus fundamentos fueron, sin excepción, impugnados judicialmente en el Perú. Las actuaciones judiciales concluyeron con pronunciamientos favorables a la segunda demandante.

Las partes demandantes sostienen que esos procesos judiciales se resolvieron definitiva e irrevocablemente conforme a la legislación peruana, y tienen ahora el carácter de cosa juzgada. Ninguna entidad pública o privada intentó siquiera impugnar esas sentencias judiciales ${ }^{39}$. Además, alegan las partes demandantes que la construcción de la fábrica se hizo bajo el estricto cumplimiento de la normativa ambiental y respetando el ecosistema de la zona, según el estudio de impacto ambiental que presentaron previo a la construcción de la fábrica y que fue aprobado por el Instituto Nacional de Recursos Naturales (INRENA), entidad estatal peruana competente en materia ambiental, dependiente del Ministerio de Agricultura y que demostró la inexistencia de contaminación ambiental alguna ${ }^{40}$.

En el 1999, la Municipalidad de Chorrillos concedió a la segunda demandante su licencia operacional y después de desarrollar su actividad inversora sin que se presentase obstáculo alguno, en el año 2001, la mencionada autoridad peruana revocó la licencia otorgada, alegando la necesidad pública de preservación, mantenimiento y defensa de la Reserva Ecológica Pantanos de Villa, zona en la que se desarrolló la inversión, motivos por los cuales, se le encomendó al Alcalde de Lima presentar al Parlamento peruano un proyecto de ley de expropiación por razones de necesidad pública ${ }^{41}$. Las Demandantes sostienen que la Demandada infringió las obligaciones contenidas en los artículos del APPRI, específicamente aquellos relacionados con la protección conforme a la ley y frente a medidas injustificadas o discriminatorias, las garantías de un tratamiento justo y equitativo, nacional y de nación más favorecida y protección frente a expropiaciones ilegales, discriminatorias o sin compensación ${ }^{42}$.

La parte demandada, presentó tres excepciones respecto a la jurisdicción del tribunal, consistentes en: i) incompetencia ratione temporis, las disposiciones del APPRI no se aplican a diferencias y controversias surgidas antes de que entre en vigencia dicho tratado; ii) falta de competencia por presentación previa 
de la controversia ante los tribunales locales; iii) incompetencia ratione materiae, el APPRI solo se aplica a "inversiones" realizadas conforme a las disposiciones legales de la otra parte contratante. Las partes demandantes negaron todos los argumentos presentados por Perú, argumentando que la controversia, refiriéndose a la originada a partir del año 2001, era distinta a la controversia surgida en los años noventa y que ya se había resuelto ante los órganos judiciales peruanos.

En efecto, el Gobierno peruano contra-alegó que los fallos judiciales dirigidos a la concesión de los permisos fueron obtenidos mediante corrupción, por actos ilegales, mediante la presentación de pruebas falsas por parte de oficiales de la administración gubernamental anterior, por lo que no existía jurisdicción bajo el Tratado Bilateral de Inversión (TBI. El tribunal arbitral determinó carecer de jurisdicción, puesto que los hechos en controversia antecedieron al propio TBI. También reconoció que, de existir prueba de la corrupción alegada por Perú, la misma hubiese constituido un fundamento adicional para determinar falta de jurisdicción ${ }^{43}$.

Lo ocurrido en los casos analizados justifica una correcta actuación en el arbitraje internacional, no solo por parte de los inversores, sino también de los Estados y, en particular, de sus instituciones públicas al asumir una alta responsabilidad gubernamental en sus actuaciones. Responsabilidad gubernamental que condiciona, a nuestro juicio, la existencia de una presunción a favor de una mejor protección del interés público y, consigo, una mayor transparencia procesal en el arbitraje internacional, teniendo en cuenta que tanto los ciudadanos como terceros ajenos al procedimiento, en determinadas circunstancias, pueden tener un interés legítimo ${ }^{44}$ en conocer e informarse, sobre el litigio y el procedimiento arbitral. Estos casos nos demuestran que tanto los demandantes, coincidente siempre con el inversor, y los demandados con los Estados anfitriones o receptores de la inversión, enfrentan desde diferentes perspectivas el actuar ilícito de ciertos Estados en el ámbito de las inversiones.

Mientras que el inversor tiene un interés en combatir la corrupción de los gobiernos con el objetivo de que se establezcan mercados justos y predecibles para sus operaciones internacionales, corriendo el riesgo en el futuro que le pueda afectar en sus relaciones con las administraciones estatales, del otro lado nos encontramos con que los Estados asumen también una posición esencial de combatir la corrupción para fomentar y proteger la legitimidad de sus instituciones gubernamentales, en estos casos, desde la perspectiva del interés general, teniendo en cuenta que la existencia de estos actos ilícitos pueden ocasionar posibles incumplimientos de las obligaciones internacionales contraídas por los Estados.

Además, ningún Estado estaría interesado en que se emita un laudo en su contra en un procedimiento arbitral y que el mismo se fundamente en la existencia de ciertos actos de corrupción. En este sentido, no solo vería empañada su imagen a nivel internacional, sino que crearía un precedente negativo de sus propias actuaciones. Al mismo tiempo, las violaciones de los estándares requeridos por los Estados anfitriones pueden materializarse en efectos negativos directos o indirectos ejercidos sobre el inversor extranjero o su actividad inversora ${ }^{45}$, lo que justifica la existencia de una relación equilibrada en cuanto a obligaciones y derechos entre el inversor extranjero y el Estado anfitrión.

\section{Consideraciones finales}

Los casos aquí analizados son un ejemplo de la inexcusable presencia del interés público presente en el arbitraje de inversiones, en los que se demuestra que el objeto de la litis siempre involucra cuestiones relacionadas con los derechos fundamentales de las poblaciones afectadas, primando, entre otros, las cuestiones medioambientales y de salud pública. Motivos que justifican, sin temor a equívocos, la participación de terceros amicus curiae, y el conocimiento público de las actuaciones durante todo el procedimiento. Cuestiones éstas que, sin un adecuado tratamiento de la transparencia en el procedimiento arbitral, sería imposible lograr. 


\section{Referencias}

Aguas Provinciales de Santa Fe S. A., Suez, Sociedad General de Aguas de Barcelona S. A., and Interaguas Servicios Integrales del Agua S. A. vs. Argentine Republic, ICSID Case No. ARB/03/17 (2006).

Barnali Choudhury, Recapturing Public Power: ¿Is Investment Arbitration's Engagement of the Public Interest Contributing to the Democratic Deficit?, 41 Vanderbilt Journal of Transnational Law, n. ${ }^{\circ} 784,775-832$ (2008). https://pdfs.semanticscholar.org/9c4b/90ea2ec61418c7953681484a524b9f879178.pdf

BIVAC vs. República del Paraguay, Caso ICSID ARB/07/9 (2009).

Caroline Henckels, Public-Private Arbitration in Australia: Public Law Concerns, Private Law Responses, en The Comparative Constitutional Law of Private-Public Arbitration (Oxford University Press, 2018). https://ssrn.c om/abstract $=3013518$

Centro Internacional de Arreglo de Diferencias Relativas a Inversiones (CIADI), 2019 Informe Annual Excelencia en la resolución de diferencias relativas a inversiones (2019). https://icsid.worldbank.org/sites/default/files/public ations/annual-report/es/ICSID_AR19_Spanish_CRA_Web_Low_DD.pdf

Comisión de las Naciones Unidas para el Derecho Mercantil Internacional (CNUDMI). Reglamento de la CNUDMI sobre la Transparencia en los Arbitrajes entre Inversionistas y Estados en el Marco de un Tratado (2014). https:/ /www.uncitral.org/pdf/spanish/texts/arbitration/rules-on-transparency/Rules-on-Transparency-S.pdf

Daniel Barstow Magraw Jr. \& Niranjali Manel Amerasinghe, Transparency and Public Participation in Investor-State Arbitration, 15 Journal of International and Comparative Law, n. ${ }^{\circ}$ 2, 337-360 (2009). https://nsuworks.nova.e $\mathrm{du} / \mathrm{ilsajournal} / \mathrm{vol} 15 /$ iss $2 / 4 /$

Dimitrios Katranstiotis, Transparency in International Investment Arbitration: From the Current towards the Future Normative Framework (2014) (tesis doctoral, International Hellenic University). https://repository.ihu.edu.gr/xmlui/bitstream/handle/11544/454/Dimitrios\%20Katrantsiotis_5 0582_assignsubmission_file_Dissertaion-Dimitrios\%20Katrantsiotis.pdf?sequence $=1$

EDF (Services) Limited vs. Rumania, Caso CIADI No. ARB/05/13, Laudo (2009).

Empresas Lucchetti, S. A. (Chile) y Lucchetti Perú, S. A. (Perú) vs. República del Perú, caso CIADI, No. ARB/03/4 (2002).

Epaminontas E. Triantafilou, Is a Connection to the "Public Interest" a Meaningful Prerequisite of Third Party Participation in Investment Arbitration, 5 Berkeley Journal of International Law Publicist, 38-46 (2010). https: //bjil.typepad.com/triantafilou_macro.pdf

Filip Balcerzak, Jarrod Hepburn, Publication of Investment Treaty Awards: The Qualified Potential of Domestic Access to Information Laws, 3 Groningen Journal of International Law, n. ${ }^{\circ}$ 1, 147-170 (2015). https://doi.org/10.218 27/5a86a8b976e29

Fraport AG Frankfurt Airport Services Worldwide vs. República de las Filipinas, caso CIADI, No. ARB/03/25 (2003).

F-W Oil Interests, Inc. (EEUU) vs. República de Trinidad \& Tobago, caso CIADI, No. ARB/01/14 (2001).

Gábor Szalay, Arbitration and Transparency-Relations Between a Private Environment and a Fundamental Requirement (2016) (tesis de maestría, Tilburg University). http://arno.uvt.nl/show.cgi?fid=142480

Juan Fernández-Armesto, La lucha contra la corrupción desde el arbitraje, VII Conferencia Internacional Hugo Grocio de Arbitraje (CEU Ediciones, 2018).

Julie A. Maupin, Transparency in International Investment Law: The Good, the Bad and the Murky, en Transparency in International Law, 142-171 (Andrea Bianchi \& Anne Peters eds., Cambridge University Press, 2013). https:/ /scholarship.law.duke.edu/cgi/viewcontent.cgi?article $=5840 \&$ context $=$ faculty_scholarship

Metal-Tech Ltd. vs. República de Uzbekistán, Caso CIADI ARB/10/3 (2010).

Methanex Corp vs. United States of America, Decision on petitions from third persons to intervene as "amici curiae" (2001). https://www.iisd.org/pdf/methanex_tribunal_first_amicus_decision.pdf

Methanex Corp vs. United States of America, In the Matter of an Arbitration Under Chapter 11 of the North American Free Trade Agreement and the UNCITRAL Arbitration Rules (1999). https://www.italaw.com/cases/683 
Minutes of order of the second procedural meeting held at the World Bank, Washington DC (2000). https://www.i talaw.com/sites/default/files/case-documents/italaw9094.pdf

N. Jansen Calamita, Dispute Settlement Transparency in Europe's Evolving Investment Treaty Policy, 15 The Journal of World Investment \& Trade, n. 3-4, 645-678 (2014). https://doi.org/10.1163/22119000-01504014

Nayiber Febles Pozo, Los derechos humanos vs. el CIADI: el binomio imperfecto, en Los derechos fundamentales: Perspectiva comparada entre América y Europa, 286-293 (Nayiber Febles Pozo, Guillermo Ormazabal Sánchez, Luis F. Carrillo Pozo, María Elena Cobas Cobiella, Catalina Ediltrudis Panadero De La Cruz eds., Editorial Leyer, 2019). https://www.academia.edu/41108976/Acceso_a_la_justicia_en_recientes_codificaciones_aut\% C3\%B3nomas_de_derecho_internacional_privado_en_los_pa\%C3\%ADses_mercosure\%C3\%B1os

Piero Foresti, Laura de Carli, Others vs. The Republic of South Africa, ICSID Case No. ARB(AF)/07/01 (2010).

Ruth Teitelbaum, A Look at The Public Interest in Investment Arbitration: Is it Unique? What Should We Do About It?, 5 Berkeley Journal of International Law Publicist, 54-62 (2010). https://bjil.typepad.com/teitelbaum_final-1.pdf

SGS Société Générale de Surveillance S. A. vs. Republic of the Philippines, CIADI Caso No. ARB/02/6 (2004).

Suez, Sociedad General de Aguas de Barcelona S. A., and Vivendi universal S. A. vs. Argentine Republic, ICSID Case No. ARB/03/19 (2006).

World Duty Free Company Ltd. vs. Kenya, caso CIADI, No. ARB/00/7, Laudo (2006).

\section{Notas}

* Artículo de investigación. Este artículo es producto de la tesis doctoral del autor, la cual fue financiada por las becas FI de la Generalitat de Catalunya.

1 Dimitrios Katranstiotis, Transparency in International Investment Arbitration: From the Current towards the Future Normative Framework, 3 (2014) (tesis doctoral, International Hellenic University).

2 N. Jansen Calamita, Dispute Settlement Transparency in Europe's Evolving Investment Treaty Policy, 15 The Journal of World Investment \& Trade, n. ${ }^{\circ}$ 3-4, 645-678 (2014).

3 Una de las partes en una controversia puede tener un incentivo para revelar unilateralmente un laudo arbitral. "O la divulgación puede ser legalmente requerida, como es el caso de los requisitos de presentación de informes impuestos por los valores y las comisiones de cambio o las leyes nacionales que requieren la publicación de todos los gastos del presupuesto gubernamental (incluyendo reclamaciones legales pagadas)". Julie A. Maupin, Transparency in International Investment Law: The Good, the Bad and the Murky, en Transparency in International Law, 15 (Andrea Bianchi \& Anne Peters eds., Cambridge University Press, 2013).

$4 \quad$ Filip Balcerzak, Jarrod Hepburn, Publication of Investment Treaty Awards: The Qualified Potential of Domestic Access to Information Laws, 3 Groningen Journal of International Law, n. 1,152 (2015).

5 Piero Foresti, Laura de Carli, Others vs. The Republic of South Africa, ICSID Case No. ARB(AF)/07/01, Award (2010).

6 Piero Foresti, Laura de Carli, Others vs. The Republic of South Africa, ICSID Case No. ARB(AF)/07/01, Award, párr. 28 (2010): “On 25 September 2009, the Tribunal issued a further decision on the NDP petitions. First, the Tribunal explained the reasoning behind its 11 September decision to require the Parties to agree on and disclose to the NDPs certain redacted documents. The Tribunal noted that its decision in this regard was animated by two basic principles: (1) that NDP participation is intended to enable NDPs to give useful information and accompanying submissions to the Tribunal, but is not intended to be a mechanism for enabling NDPs to obtain information from the Parties; and (2) where there is NDP participation, the Tribunal must ensure that it is both effective and compatible with the rights of the Parties and the fairness and efficiency of the arbitral process. The Tribunal had ordered the Parties to provide the NDPs with certain redacted documents because it had taken the view that the NDPs must be allowed access to those papers submitted to the Tribunal by the Parties that are necessary to enable the NDPs to focus their submissions upon the issues arising in the case and to see what positions the Parties have taken on those issues. The calendar set by the Tribunal for the NDP submissions and the Parties' responses was set because of the Tribunal's view that the NDPs must also be given adequate opportunity to prepare and deliver their submissions in sufficient time before the hearing for the Parties to be able to respond to those submissions".

7 Piero Foresti, Laura de Carli, Others vs. The Republic of South Africa, ICSID Case No. ARB(AF)/07/01, Award, paragraph 79 (2010): “(...) the Claimants sought the Respondent's consent to discontinue the proceedings pursuant to Article 50 of the Additional Facility Rules. The Claimants argued that, although they had not been provided with full relief for their alleged injuries, they nevertheless sought discontinuance because, pursuant to a 12 December 2008 
agreement between the DMR and the Operating Companies (the 'Offset Agreement'), the Respondent had granted the Claimants' Operating Companies new order mineral rights without requiring the Claimants (...)”.

Piero Foresti, Laura de Carli, Others vs. The Republic of South Africa, ICSID Case No. ARB(AF)/07/01, Award, paragraph 80 (2010): "The Claimants also asserted that they had tried to settle the case with the Respondent, but to no avail.108 Therefore, the Claimants argued, given that they had received partial relief, and given the costs of the arbitration and current economic conditions, it was now appropriate to seek discontinuance of these proceedings".

9 Piero Foresti, Laura de Carli, Others vs. The Republic of South Africa, ICSID Case No. ARB(AF)/07/01, Award, párr. 82 (2010): "The Respondent argued in its Response that the Claimants had sought discontinuance without prejudice, leaving the Claimants free to bring the same claims at a later date and leaving all costs where they lay, and that Respondent could not agree to discontinuance on such terms (...)".

10 Piero Foresti, Laura de Carli, Others vs. The Republic of South Africa, ICSID Case No. ARB(AF)/07/01, Award, párr. 82 (2010): “(....) the Claimants informed the Respondent and the Tribunal that they were willing to agree to discontinuance with an award dismissing their claims with res judicata effect".

11 Piero Foresti, Laura de Carli, Others vs. The Republic of South Africa, Award, 2010. Decision on Discontinuance and Costs.

12 Centro Internacional de Arreglo de Diferencias Relativas a Inversiones (CIADI), 2019 Informe Annual Excelencia en la resolución de diferencias relativas a inversiones (2019).

13 Véase, el Artículo 1.4 (a) del Reglamento sobre la Transparencia de la CNUDMI.

14 Véase, Artículo 3 del Reglamento sobre la Transparencia de la CNUDMI. Esta obligación se produce al inicio del procedimiento, Artículo 2 del Reglamento sobre la Transparencia de la CNUDMI, y se extiende a una amplia gama de documentos, pero no es absoluta. El Artículo 7.1 y 7.2 del propio Reglamento, establecen varias excepciones en las que se prohíbe la divulgación de la información teniendo en cuenta el carácter de confidencial o protegida de la información.

15 Véase, Artículo 6.1 del Reglamento sobre la Transparencia de la CNUDMI.

16 Daniel Barstow Magraw Jr. \& Niranjali Manel Amerasinghe, Transparency and Public Participation in Investor-State Arbitration, 15 Journal of International and Comparative Law, n. . 2, 339 (2009).

17 Barnali Choudhury, Recapturing Public Power: iIs Investment Arbitration's Engagement of the Public Interest Contributing to the Democratic Deficit?, 41 Vanderbilt Journal of Transnational Law, n. ${ }^{\circ} 784,775-832$ (2008); Nayiber Febles Pozo, Los Derechos humanos vs. el CIADI: el binomio imperfecto, en Los derechos fundamentales: Perspectiva comparada entre América y Europa, 286-293 (Nayiber Febles Pozo, Guillermo Ormazabal Sánchez, Luis F. Carrillo Pozo, María Elena Cobas Cobiella, Catalina Ediltrudis Panadero De La Cruz eds., Editorial Leyer, 2019).

18 Methanex Corp vs. United States of America, In the Matter of an Arbitration Under Chapter 11 of the North American Free Trade Agreement and the UNCITRAL Arbitration Rules (1999).

19 Para mayor conocimiento sobre el IISD y su Petición para participar como Amicus en el caso Methanex Corp vs. United States of America, puede consultarse en: Opening the Doors, at least a little: Comment on the Amicus decisión in Methanex vs. United States. https://www.iisd.org/pdf/2001/trade_reciel_methanex.pdf

20 Methanex Corp vs. United States of America. Decision on petitions from third persons to intervene as "amici curiae" (2001); ver también Minutes of order of the second procedural meeting held at the World Bank, Washington DC (2000).

21 Methanex Corp vs. United States of America, Decision on petitions from third persons to intervene as amici curiae, párr. 49 (2001): "There is an undoubtedly public interest in this arbitration... The substantive issues extend far beyond those raised by the usual transnational arbitration between commercial parties. This is not merely because one of the DiBputing Parties is a State: there are of course disputes involving States which ace of no greater general public importance than a dispute between private persons. The public interest in this arbitration miles from its subjectmatter-, as powerfully suggested in the Petitions. There is also a broader argument, as suggested by the Respondent and Canada: The Chapter 11 arbitral process could benefit from being perceived as more open or transparent: or conversely be harmed if seen as unduly secretive. In this regard, the Tribunal's willingness to recsive amicus submissions might support the process in general and this arbitration in particular; whereas a blanket refusal could do positive harm".

22 Suez, Sociedad General de Aguas de Barcelona S. A., and Vivendi universal S. A. vs. Argentine Republic, ICSID Case No. ARB/03/19 (2006).

23 Suez, Sociedad General de Aguas de Barcelona S. A., and Vivendi universal S. A. vs. Argentine Republic, Decisión sobre jurisdicción, párr. 1-2 (2006).

24 Suez, Sociedad General de Aguas de Barcelona S. A., and Vivendi universal S. A. vs. Argentine Republic, Resolución en respuesta a la petición de transparencia y participación en calidad de amicus curiae, párr. 1 (2005).

25 Suez, Sociedad General de Aguas de Barcelona S. A., and Vivendi universal S. A. vs. Argentine Republic, Resolución en respuesta a la petición de transparencia y participación en calidad de amicus curiae, párr. 19 (2005).

26 Epaminontas E. Triantafilou, Is a Connection to the "Public Interest" a Meaningful Prerequisite of Third Party Participation in Investment Arbitration, 5 Berkeley Journal of International Law Publicist, 41 (2010). 
27 Aguas Provinciales de Santa Fe S. A., Suez, Sociedad General de Aguas de Barcelona S. A., and Interaguas Servicios Integrales del Agua S. A. vs. Argentine Republic, ICSID Case No. ARB/03/17 (2006).

28 Aguas Provinciales de Santa Fe S. A., Suez, Sociedad General de Aguas de Barcelona S. A., and Interaguas Servicios Integrales del Agua S. A. vs. Argentine Republic, Resolución en respuesta a la petición de participación como amicus curiae, párr. 1 (2006).

29 Aguas Provinciales de Santa Fe S. A., Suez, Sociedad General de Aguas de Barcelona S. A., and Interaguas Servicios Integrales del Agua S. A. vs. Argentine Republic, Resolución en respuesta a la petición de participación como amicus curiae, pár.18 (2006).

30 Aguas Provinciales de Santa Fe S. A., Suez, Sociedad General de Aguas de Barcelona S. A., and Interaguas Servicios Integrales del Agua S. A. vs. Argentine Republic, Resolución en respuesta a la petición de participación como amicus curiae, párr.18 (2006).

31 En este aspecto, se produce, según Choudhury, un "déficit democrático, cuando aquellos que se ven afectados por las normas y decisiones de un organismo internacional, no se sienten como si tuvieran una voz significativa en la creación y aplicación de esas normas"; Barnali Choudhury, op. cit., 784.

32 Julie A. Maupin, op. cit., 14.

33 Ruth Teitelbaum, A Look at The Public Interest in Investment Arbitration: Is it Unique? What Should We Do About It?, 5 Berkeley Journal of International Law Publicist, 56 (2010).

34 Véase, los casos BIVAC vs. República del Paraguay, Caso ICSID ARB/07/9. Decisión sobre las excepciones a la jurisdicción, párrafs. 7, 119-120 (2009), en relación con el contrato entre el reclamante y el Ministerio de Hacienda de Paraguay. Prestación de servicios técnicos para la inspección previa a la expedición de las importaciones en el Paraguay, alegando que el incumplimiento por parte del Ministerio de Hacienda de "cumplir con su parte del trato" constituía una violación del TBI entre los Países Bajos y Paraguay; SGS Société Générale de Surveillance S. A. vs. Republic of the Philippines, CIADI Caso No. ARB/02/6. Decisión sobre las excepciones a la jurisdicción (2004).

35 Véase los casos Fraport AG Frankfurt Airport Services Worldwide vs. República de las Filipinas, caso CIADI, No. ARB/03/25 (2003); SGS Société Générale de Surveillance S.A. (Suiza) vs. República de las Filipinas, caso CIADI, No. ARB/02/6 (2004); F-WOil Interests, Inc. (EEUU) vs. República de Trinidad \& Tobago, caso CIADI, No. ARB/01/14 (2001); Metal-Tech Ltd. vs. República de Uzbekistán, Caso CIADI ARB/10/3 (2010); EDF (Services) Limited vs. Rumania, Caso CIADI No. ARB/05/13, Laudo (2009).

36 World Duty Free Company Ltd. vs. Kenya, caso CIADI, No. ARB/00/7, Laudo (2006). Este caso ha resultado ser tan significativo en la práctica arbitral, que el árbitro internacional Fernández-Armesto lo ha nombrado como "un caso paradigmático"; Juan Fernández-Armesto, La lucha contra la corrupción desde el arbitraje, VII Conferencia Internacional Hugo Grocio de Arbitraje, 20-21 (CEU Ediciones, 2018).

37 Empresas Lucchetti, S. A. (Chile) y Lucchetti Perú, S. A. (Perú) vs. República del Perú, caso CIADI, No. ARB/03/4 (2002).

38 Empresas Lucchetti, S. A. (Chile) y Lucchetti Perú, S. A. (Perú) vs. República del Perú, Laudo, párr. 3 (2005).

39 Empresas Lucchetti, S. A. (Chile) y Lucchetti Perú, S. A. (Perú) vs. República del Perú, Laudo, párr. 18 (2005).

40 Empresas Lucchetti, S. A. (Chile) y Lucchetti Perú, S. A. (Perú) vs. República del Perú, Laudo, párr. 19 (2005).

41 Empresas Lucchetti, S. A. (Chile) y Lucchetti Perú, S. A. (Perú) vs. República del Perú, Laudo, párr. 20 (2005).

42 Empresas Lucchetti, S. A. (Chile) y Lucchetti Perú, S. A. (Perú) vs. República del Perú, Laudo, párr. 23 (2005).

43 Empresas Lucchetti, S. A. (Chile) y Lucchetti Perú, S. A. (Perú) vs. República del Perú, Laudo, párr.48-62 (2005).

44 Caroline Henckels, Public-Private Arbitration in Australia: Public Law Concerns, Private Law Responses, en The Comparative Constitutional Law of Private-Public Arbitration (Oxford University Press, 2018).

45 Gábor Szalay, Arbitration and Transparency-Relations Between a Private Environment and a Fundamental Requirement, 21 (2016) (tesis de maestría, Tilburg University).

\section{Licencia Creative Commons CC BY 4.0}

Cómo citar este artículo: Nayiber Febles Pozo, La transparencia y el interéspúblico en el arbitraje de inversiones, 69 Vniversitas (2020). https://doi.org/10.11144/Javeriana.vj69.tiai 